\title{
A Framework for Controlling electric car via Brain-Computer Interfaces
}

\author{
Jinghai Yin, Jianfeng $\mathrm{Hu}$, Zhendong Mu \\ Institute of Information Technology, Jiangxi University of Technology, Nanchang 330098, China
}

Keywords: Brain-Computer Interface; Electric Cars; EEG

\begin{abstract}
To enhance human interaction with machines, research interest is growing to develop a "Brain-Computer Interface" (BCI), which allows communication of a human with a machine only by use of brain signals. In this paper, one type of application was designed for controlling electric car by BCI. The application is based on a flexible software framework. The BCI system can make for the disabled to control the operation of electric cars through movement imaginary.
\end{abstract}

\section{Introduction}

EEG based brain computer interface (BCI) systems can be used for people with disabilities to improve their quality of life. Applications of BCI systems comprise the restoration of movements, communication and environmental control [1]. The most challenging thing in these applications is to control cars by human's EEG. A few of research has been performed on create information chain from brain to cars by BCI system. DuanFeng and his research team of Nankai university implements communication between auto control system and the EEG signals, the equipment can control the car by thoughts of human.

Although some progress has been made in this area, at least two major obstacles must be overcome before BCI technology has begun to develop commercial applications. Firstly, most of BCI systems were achieved under laboratory conditions, lack of flexibility, scalability, and availability. Secondly, full set of BCI system equipment was both complex and expensive, and the related applications were difficult to promotion. The above difficulties are real challenges faced by researchers attempting to develop. In recent years, with the popularity of electric cars, more and more people choose to use it as transportation. At the same time it also has the advantages of simple operation, energy conservation and environmental protection. The brain-machine interface technology used in electric vehicle drive control system, will make it easier for action of persons with disabilities to travel alone to become a possibility.

The goal of this paper is to design a framework for controlling electric car on real-time BCI system that includes physiological signal acquisition, EEG transmission, and a mobile devices which be used to analyze and process EEG and then control cars.

\section{System Architecture}

The Architecture diagram of the Pocket PC game based BCI system is shown in Fig. 1, which includes four units: Dedicated EEG Cap; signal acquisition and amplification unit; Signal Processing module and controller. The four units and driver constitute a complete data chain. 


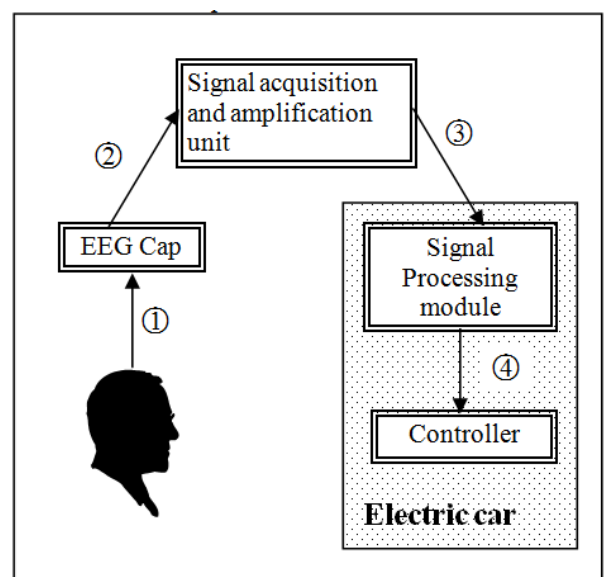

Figure 1. Architecture of electric car BCI System

There are four steps in this data cycle. 1) Human's thinking activity will make the changes in cortical potentials. This change can be acquisition by The EEG cap which can acquire this change by high sensitivity electrode. 2) This signal is send to Signal acquisition and amplification unit by data cable of EEG cap. The device supports 8 analog input channels digitized at 16 bit resolution and sampled at a fixed $256 \mathrm{~Hz}$ sampling rate. 3) By Signal acquisition and amplification unit, the original signal is transform to formatted data like a huge data matrix. 4) The main function of this unit is to transform pretreatment EEG signal into control signal like turn left, turn right, forward, backward, stop etc. The driver will get feedback by the car's movement.

\section{Requirements Analysis}

The first things we need to do is the requirements analysis before the software developing, UML as a common modeling tools have many types of diagrams. One of the most important UML diagrams is use case diagram. The following figure 2 is the use case diagram of $\mathrm{BCI}$ system for electric car:

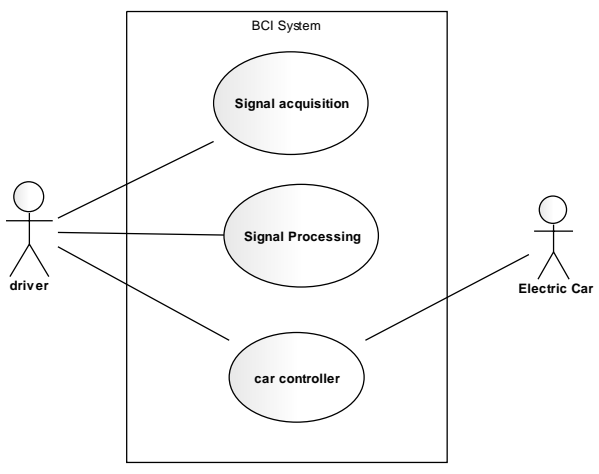

Figure 2. Use case diagram of electric car BCI System

As figure 2 shows, there are three main use cases, signal acquisition, signal processing and car controller. Signal acquisition use case is responsible for read EEG data packets from EEG acquisition device by Bluetooth port, and then send to signal processing module after data formatting. Signal processing use case is responsible for analysis and processing EEG data from the signal analysis modules, the last results will be sent to car controller. Finally car controller produce control signals to 
control the movement direction of the electric car and speed. The two roles in the figure are the driver and electric car.

Figure 3 and figure 4 are two activity diagrams of signal acquisition use case and signal processing use cases.

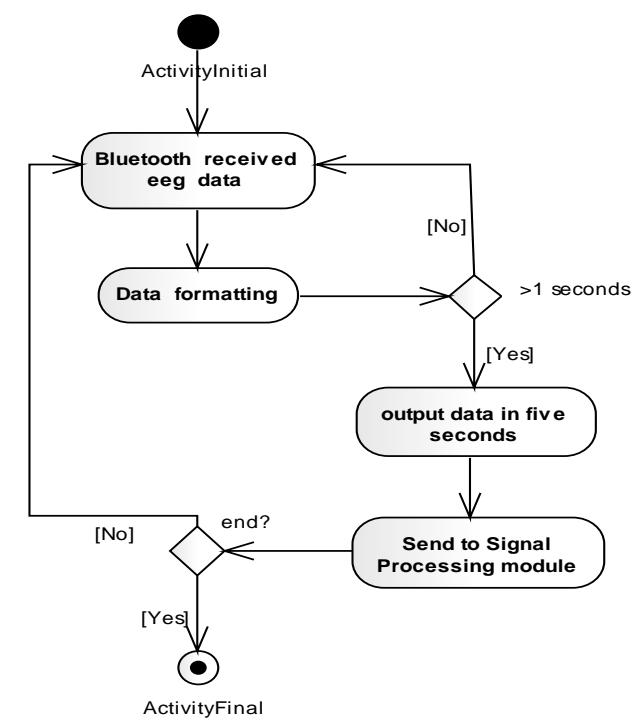

Figure 3. Activity diagram of signal acquisition

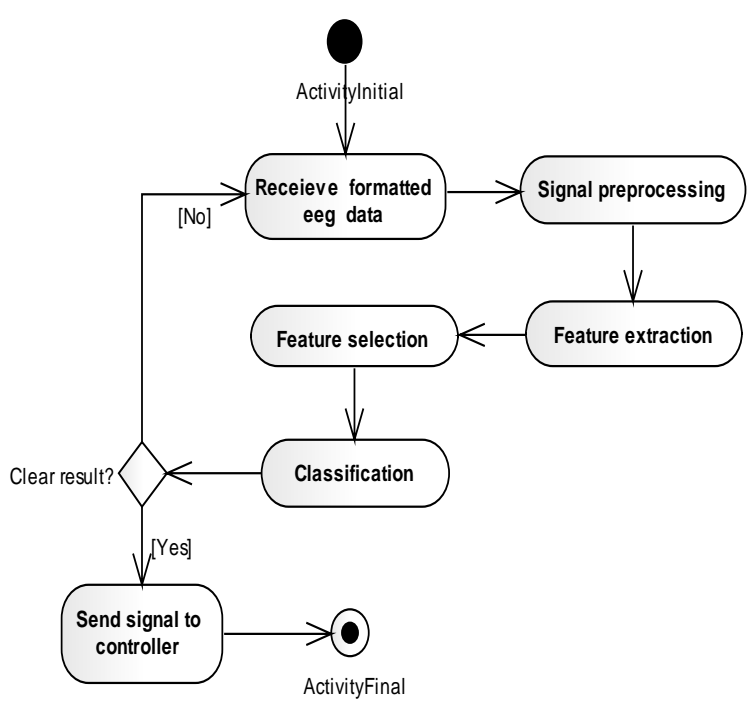

Figure 4. Activity diagram of signal processing

It can be seen from figure 3 that system receive the EEG data via Bluetooth port firstly, and then storage data after formatting, the next step it determine whether more than one second, if there is no more than one second continues to read the data, if more than one second it will output EEG data collected in five seconds, and finally sent it to the signal processing module.

From figure 4 we can seen that the system will receive the formatted EEG data sent from acquisition module. And then system through four steps such as signal preprocessing, feature extraction, feature selection, classification. If certain classification results were obtained the system will send signals to the controller, or to give up the analysis results, continue to the next data analysis.

\section{Framework Architecture}

When developing the software architecture for the interaction framework, we tried to adhere to a simple class structure. The Class diagram captures the logical structure of the system: the Classes - including Active and Parameterized Classes - and things that make up the model. It is a static model, describing what exists and what attributes and behavior it has, rather than how something is done. Class diagrams are most useful to illustrate relationships between Classes and Interfaces. The class structure of electric car BCI system can be found in Fig. 5. 


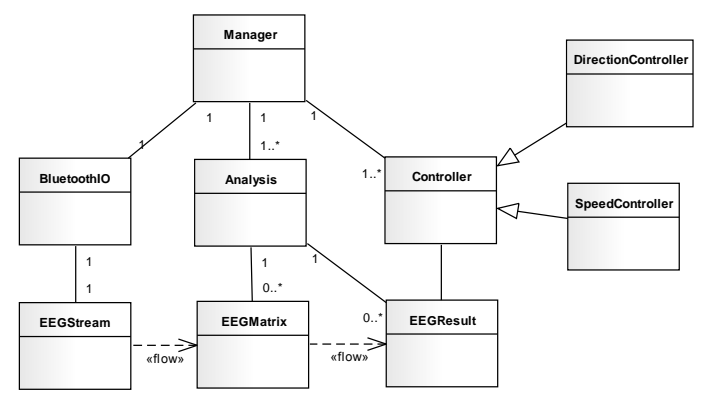

Figure 5. Architecture of electric car BCI System

In the figure there are three data entity classes including EEGStream, EEGMatrix, EEGResult. EEGStream class is responsible for electrical streaming data storage and management, EEGMatrix class is responsible for electrical matrix data storage and management, this is the standard format of EEG data, while EEGResult class is responsible for the storage of non-standard matrix data format of the identification results of all kinds of intermediate results and final. Action classes contain Bluetooth, Analysis, and the Controller, etc. Bluetooth class is responsible for communication with Bluetooth port and management, Analysis class is responsible for all kinds of signal Analysis and processing method calls and management, the classification of the Controller class will receive the results into a control signal is sent to the electric car, it has two subclasses, respectively is DirectionController classes and SpeedController class. All the operation process be unified managed by manager class.

\section{Discussion}

The framework presented in this paper enables the flexible and easy definition and test of interaction concepts at runtime. The BCI provides a large set of possible interactions that can be used for controlling an application. However, the carrier of the BCI may prefer some interactions over others. Our framework supports individual storage of user preferences. These settings can be changed and modified at runtime. Achievement of these will be used widely for patients who suffer from severe motor impairments and will perfect technological foundation for future commercial application.

\section{Acknowledgment}

This work was supported by Jiangxi province department of science and technology support project [20142bbe50030] and Natural Sciences Project of Jiangxi Science and Technology Department [20122BAB201049]. The authors are grateful for the anonymous reviewers who made constructive comments.

\section{References}

[1]G. Pfurtscheller, C. Guger, G. Müller, G. Krausz, and C. Neuper, Brain oscillations control hand orthosis in a tetraplegic," Neurosci. Lett., vol. 292, pp. 211-214, $2000 .$.

[2]Ince N.F., Arica S., Tewfik A., "Classification of single trial motor imagery EEG recordings with subject adapted nondyadi arbitrary time-frequency tilings," J Neural Eng, Vol. 3, pp. 235-244, 2006. 
Jianfeng Hu,Dan Xiao,Zhendong Mu. Application of Energy Entropy in Motor Imagery EEG Classification. International Journal of Digital Content Technology and its Applications,2009,3(2): 83-90

[3]R. Nicole, "Title of paper with only first word capitalized," J. Name Stand. Abbrev., in press.

McFarland D.J., Wolpaw J.R., "Sensorimotor rhythm-based brain-computer interface (BCI): model order selection for autoregressive spectral analysis," J Neural Eng, Vol. 5, pp. 155-162, 2008.

[4]Ramoser H., Müller-Gerking J., Pfurtscheller G., "Optimal spatial filtering of single trial EEG during imagined hand movement," IEEE Trans Rehabil Eng, Vol. 8, pp. 441-446, 2000.

[5]Müller-Gerking J., Pfurtscheller G., Flyvbjerg H., "Designing optimal spatial filters for single-trial EEG classification in a movement task," Clin Neurophysiol, Vol. 110, pp. 787-798, 1999. 